\title{
Efficacy of Extracorporeal Shockwave Therapy on Cervical Myofascial Pain Following Neck Dissection Surgery: A Randomized Controlled Trial
}

\author{
FatmaAlzahraa Hassan Kamel, $\mathrm{PhD}^{1,2}$, Maged Basha, $\mathrm{PhD}^{1}$, Ashwag Alsharidah, $\mathrm{MD}^{3}$, \\ Islam Mohamed Hewidy, $\mathrm{PhD}^{4}$, Mohamed Ezzat, $\mathrm{PhD}^{5}$, Nancy Hassan Aboelnour, $\mathrm{PhD}^{2}$ \\ ${ }^{1}$ Department of Physical Therapy, College of Medical Rehabilitation, Qassim University, Buraidah, Saudi Arabia; \\ ${ }^{2}$ Department of Physical Therapy for Surgery, Faculty of Physical Therapy, Cairo University, Cairo, Egypt; \\ ${ }^{3}$ Department of Physiology, College of Medicine, Qassim University, Buraidah, Saudi Arabia; \\ ${ }^{4}$ Department of Physical Therapy, New York Medical College, Valhalla, NY, USA; \\ ${ }^{5}$ Department of Physical Therapy for Orthopedics, Faculty of Physical Therapy, Cairo University, Cairo, Egypt
}

Objective To investigate the efficacy of extracorporeal shockwave therapy (ESWT) on cervical myofascial pain following neck dissection in reducing pain and improving cervical range of motion (ROM).

Methods Forty-six patients with cervical myofascial pain following neck dissection surgery were recruited and subdivided at random into two equal groups. The ESWT group received ESWT once a week for $4 \mathrm{weeks}\left(0.25 \mathrm{~mL} / \mathrm{mm}^{2}\right.$, 1,000 shocks) and a topical non-steroidal anti-inflammatory drug (3 times/day for 4 weeks). The control group received only topical NSAID. The pain assessment was done by using the visual analog scale (VAS) and pressure algometry. A cervical ROM device was used for the assessment of the lateral flexion and rotation of the neck ROM on both sides. All measurements were collected at baseline, 2 weeks, and 4 weeks.

Results The ESWT group revealed a significant improvement in all parameters at post I and post II than did the control group ( $p>0.001)$, that revealed a statistical decrease only in the VAS score at post I without any statistical difference in the pain threshold and neck ROM. However, there were statistical differences in all parameters at post II compared to those at pre-treatment and post I $(\mathrm{p}<0.001)$.

Conclusion As a confirmation of the efficacy of ESWT in cervical myofascial pain control following neck dissection, we observed better results with no side effects in the ESWT group (Clinical Trial Registry No. PACTR202002648274347).

Keywords Myofascial pain syndromes, Neck dissections, Extracorporeal shock wave therapy, Visual analog scales, Pressure algometry

Received March 25, 2020; Revised May 12, 2020; Accepted July 6, 2020; Published online September 28, 2020

Corresponding author: Nancy Hassan Aboelnour

Department of Physical Therapy for Surgery, Faculty of Physical Therapy, Cairo University, 7th Ahmed Elzayat St., Bein Al Sarayat, Giza 12613, Egypt. Tel: +20-10-68904389, Fax:+20-23-761-7692, E-mail: nancyabolnour@hotmail.com

ORCID: FatmaAlzahraa Hassan Kamel (https://orcid.org/0000-0003-1546-6666); Maged Basha (https://orcid.org/0000-0002-3422-6193); Ashwag Alsharidah (https://orcid.org/0000-0003-0923-7525); Islam Mohamed Hewidy (https://orcid.org/0000-0001-5778-9039); Mohamed Ezzat (https:// orcid.org/0000-0002-7122-4621); Nancy Hassan Aboelnour (https://orcid.org/0000-0003-1643-0606).

(c) This is an open-access article distributed under the terms of the Creative Commons Attribution Non-Commercial License (http://creativecommons.org/ licenses/by-nc/4.0) which permits unrestricted noncommercial use, distribution, and reproduction in any medium, provided the original work is properly cited. Copyright (c) 2020 by Korean Academy of Rehabilitation Medicine 


\section{INTRODUCTION}

The surgical dissection of the neck is a typical technique for the management of head and neck malignant growths from the squamous cell carcinoma [1]. There are various approaches to performing the neck dissection surgery. It is worth noting that each surgical approach involves different anatomical structures and has associated postoperative side effects. One of these approaches is the radical neck dissection that involves the total excision of lymph nodes from one side of the neck, the muscle that helps turn the head (sternocleidomastoid muscle), a significant vein (internal jugular vein), and a nerve that is essential in the full-range of movement of the arm and shoulder (spinal accessory nerve). In contrast, the modified radical neck dissection preserves one or more of the aforementioned important structures [2,3]. Furthermore, selective neck dissections are performed to preserve important anatomical structures, while safely excising cancerous lymph nodes from the neck tissues on the affected side [3]. Morbidities related to neck and shoulder surgeries for head and neck cancer treatment are a major point of concern. Van Wilgen et al. [4] showed that shoulder and neck pain can affect the patients' quality of life at least one year following neck dissection. Early postoperative complications include infection, thrombosis, chyle leak, and cardiac problems. The most widely recognized late complications include neck pain and stiffness, limited shoulder and cervical range of motion (ROM), lymphedema, a reduction in mouth opening, and swallowing difficulty. While a radical neck dissection produces the greatest changes in neck and shoulder function, a selective neck dissection produces the least [5].

The incidence of neck morbidity following cancer treatment was significant and prominent including neck pain, the lack of sensation, and restricted ROM [4]. In an investigation of 25 patients with permanent neck pain following neck dissection, two types of neck pain were described; neuropathic and myofascial pains [6]. Moreover, in 220 patients who underwent neck dissection surgery, neck and shoulder pains were observed in $33 \%$ and $37 \%$ of the patients, respectively, while neuropathic and myofascial pains were observed in $32 \%$ and $46 \%$, respectively [4].

Myofascial pain syndrome (MPS) is a common musculoskeletal disorder characterized by an intensive and deep pain in one or more muscles and fasciae besides muscle tightness, tender points, limited ROM, fatigue, weakness and occasionally autonomic dysfunction $[7,8]$. MPS can be diagnosed by using pressure algometry for pain threshold evaluation [9]. Physical examination yields a palpable intramuscular band tension, muscle tenderness, and trigger points causing localized or referred pain [10]. Myofascial pain can be managed pharmacologically using non-steroidal anti-inflammatory drugs, antidepressants, anticonvulsants, and muscle relaxants; and non-pharmacologically using dry needling, trigger point injection (TPI), transcutaneous electrical nerve stimulation (TENS), and ultrasound (US) [11].

Extracorporeal shockwave therapy (ESWT) has been introduced efficiently for more than 20 years as a treatment modality in orthopedic and musculoskeletal disorders such as plantar fasciitis, calcific tendinitis, non-union of bone fractures, and epicondylitis [12-14]. ESWT has mechanical and cellular impacts on tissue regeneration and pain management through cavitation bubbles, acoustic microstreaming, and hypervascularity that can directly affect tissue calcifications, thereby modulating cell activity. Pain management can be achieved by blockage of the gate control mechanism through firing of the large sensory nerve fibers (A $\beta$ fibers) in the affected site, which in turn inhibits pain through GABAergic interneurons in the dorsal horn. The vicious cycle of MPS (pain-spasmischemia-pain) can be inhibited by the mechanical resolution of persistent sarcomere contractures, through the localized stretching of the sarcomeres by applying shockwaves vertical to the fiber alignment until the abnormal sarcomere knots are destroyed. The energy generated during the process, excessive tissue perfusion, and angiogenesis can reduce the resultant ischemia to eliminate vasoneuroactive substances released by the pressure applied on the tissue [14-25].

Minimal evidence exists regarding the efficacy of ESWT on MPS after surgery; therefore, this study strived to examine the efficiency of ESWT as a mono physiotherapeutic modality in MPS management following a neck dissection surgery, in term of improving pain and cervical ROM. This study may provide a safe, effective and noninvasive treatment modality for MPS, in an attempt to avoid invasive modalities and oral medications that cannot be tolerated for a long period due to their systemic adverse effects. 


\section{MATERIALS AND METHODS}

\section{Subjects}

Forty-six patients diagnosed with cervical MPS following a neck dissection surgery were referred to our outpatient clinic from the National Cancer Institute, Cairo University, and participated in this study. The diagnosis of MPS was done by an orthopedic specialist according to Simons' criteria [26], where five major criteria and a minimum of one to three minor criteria are required to confirm diagnosis. The major criteria were: (1) localized neck pain, (2) pain or altered sensation within the anticipated allocation of referred pain from myofascial tender points, (3) patients with palpable intramuscular taut bands, (4) oversensitive spot tenderness at one point on the length of the tense band, and (5) restriction in cervical ROM. The minor criteria were: (1) provocation of the clinical symptoms through active trigger point compression, (2) the presence of a local twitch reaction by snapping the trigger point or inserting a needle inside the trigger point within the tense band, and (3) pain relief by muscle lengthening or stretching.

Patients were enrolled in the trial if they met the following criteria: (1) age ranging from 35-60 years, (2) previous unilateral modified radical neck dissection, (3) a history of MPS involving upper trapezius for at least 3 months, and (4) moderate to severe pain (VAS score $>4$ ). The exclusion criteria were: (1) patients who received medication or other therapies for MPS within the previous 2 months, (2) the presence of a pacemaker, (3) pregnancy, (4) a wound in the affected area, (5) a cervical disk lesion, myelopathy or radiculopathy, (6) a cervical spine fracture or spondylolisthesis, (7) rheumatoid arthritis, (8) coagulopathy, and (9) epilepsy or any psychological disorders.

\section{Sample size determination}

Pre-trial sample size computation was done via G*Power statistical software (version 3.1.9.2; https://www.gpower. hhu.de/)—F tests, MANOVA (repeated measurements and between factors), $\alpha=0.05, \beta=0.2$, Pillai $V=0.16$, and large effect size, revealing the convenient sample size to be $\mathrm{n}=46$ [27].

\section{Design}

This trial was a single-blinded randomized controlled experimental study and was assented by the Ethical Com- mittee of the Faculty of Physical Therapy, Cairo University (No. P.T.REC/012/001857). The study was retrospectively registered in the Pan African Clinical Trials Registry (No. PACTR202002648274347). All aspects of the study were disclosed and informed consent was obtained. The patients were randomly assigned into two equal groups via the envelope mode. After patients' agreement to participate in the study, cards with either "ESWT" or "topical diclofenac gel" recorded on them were closed in envelopes; then a blinded physical therapist was asked to select one envelope. According to the selected card, patients were assigned to their corresponding group. Dates for starting the allocated therapy were regulated and the therapy was begun after the first week of randomization. The examiner physical therapist was not included in randomization procedures and was unaware of the therapy allocation. Patients were asked not to disclose their therapy allocation to the physical therapist during assessment. The participants were informed to report any harmful effects throughout the treatment period.

\section{Assessment methods}

\section{VAS}

The VAS was used for assessing and quantifying pain exhibited by the patients through a 0-10 scale. Each subject was informed to mark the line according to the pain intensity experienced at that particular time where 0 means no pain and 10 means worst conceivable pain.

\section{Cervical ROM assessment}

The cervical ROM device (CROM; Performance Attainment Associates, Lindstrom, MN, USA) was used for the assessment of the neck lateral flexion and rotation on both sides. The CROM instrument contains a plastic frame put on the head above the nose and ears, attached to the occiput by a Velcro strap to allow for precise measurement, and it eliminates individual errors. Two independent inclinometers were connected to the frame and pointed the head position in relation to the gravity line. Another inclinometer indicated the head position in rotation in relation to a reference position.

\section{Pressure pain threshold assessment}

A pressure algometer (Somedic AB, Farsta, Sweden) was used for pressure pain threshold (PPT) assessment on the trigger spots. It contains a rubber disk ( $1 \mathrm{~cm}^{2}$ surface), 
attached to a pressure pole and inserted into a gauge, for recording the pressure in kilograms. Participants were informed to elucidate when the pressure sensation became painful and to immediately release the pressure. The most painful point on the upper trapezius, as reported by the patient and investigated by the algometer, was selected for PPT measurement. This point was marked by a waterproof marker on the initial visit. The average of three measurements was recorded.

\section{Treatment}

Both groups received $1 \%$ topical diclofenac gel on the tender points 3 times/day for 4 weeks. The experimental group additionally received ESWT (Duolith SD1 device; Storz medical, Tagerwilen, Switzerland) with the following parameters: 1,000 shocks; energy flux density (ED) of $0.25 \mathrm{~mL} / \mathrm{mm}^{2}$, on the trigger point area, once a week for 4 weeks. All patients were instructed not to take any other medicaments for MPS during the treatment period. All patients were evaluated at baseline, and on the 2nd and 4th weeks after initiation of therapy.

\section{Statistical analysis}

Characteristics between groups were evaluated using the descriptive and t-test, while comparing of gender and affected side distribution was done by the chi-squared test. Shapiro-Wilk test was applied for all variables for checking of normal data distribution. Homogeneity testing between groups was done by Levene's test. The comparison of the treatment impacts on rotation and side bending, ROM, VAS, and PPT within and between the groups was done by the mixed MANOVA. Partial etasquared was deemed as the effect size. Post-hoc tests utilizing Bonferroni correction were used for various subsequent comparisons. The significance level of all measurable tests was set at $\mathrm{p}<0.05$. All statistical analyses were applied by the Statistical Package for the Social Studies (SPSS) version 22 for Windows (IBM, Armonk, NY, USA).

\section{RESULTS}

Fig. 1 details the process of patient randomization in this study. Fifty-one participants were admitted and examined for eligibility; 5 of them were excluded as they did not fulfill the required criteria, whereas 46 subjects were found to be qualified to partake in the trial. Fortythree patients went through the treatment program, while 3 patients withdrew because of cancer recurrence.

\section{Subject characteristics}

The meanstandard deviation of subjects' characteristics in both groups are illustrated in Table 1. There were no statistical differences ( $p>0.05)$ in the age, weight, height, sex distribution, time from surgery, onset of pain, and diagnosis for surgery between groups.

\section{Effect of treatment on rotation and side bending ROM, VAS, and PPT}

Mixed MANOVA showed a significant interaction between treatment and time (Wilks' $\lambda=0.01, \mathrm{~F}_{(12,30)}=227.55$, $\left.p=0.001, \eta^{2}=0.98\right)$. There was a significant main effect of time (Wilks' $\lambda=0.005, \mathrm{~F}_{(12,30}=489.27, \mathrm{p}=0.001, \eta^{2}=0.99$ ). There was a significant main effect of treatment (Wilks' $\left.\lambda=0.03, \mathrm{~F}_{(6,36)}=162.55, \mathrm{p}=0.001, \eta^{2}=0.96\right)$. Tables 2 and 3 showed the descriptive statistics of rotation and side bending ROM, VAS, and PPT; and the statistical level of comparison between groups; as well as significance level of comparison between pre, post I, and post II in each group.

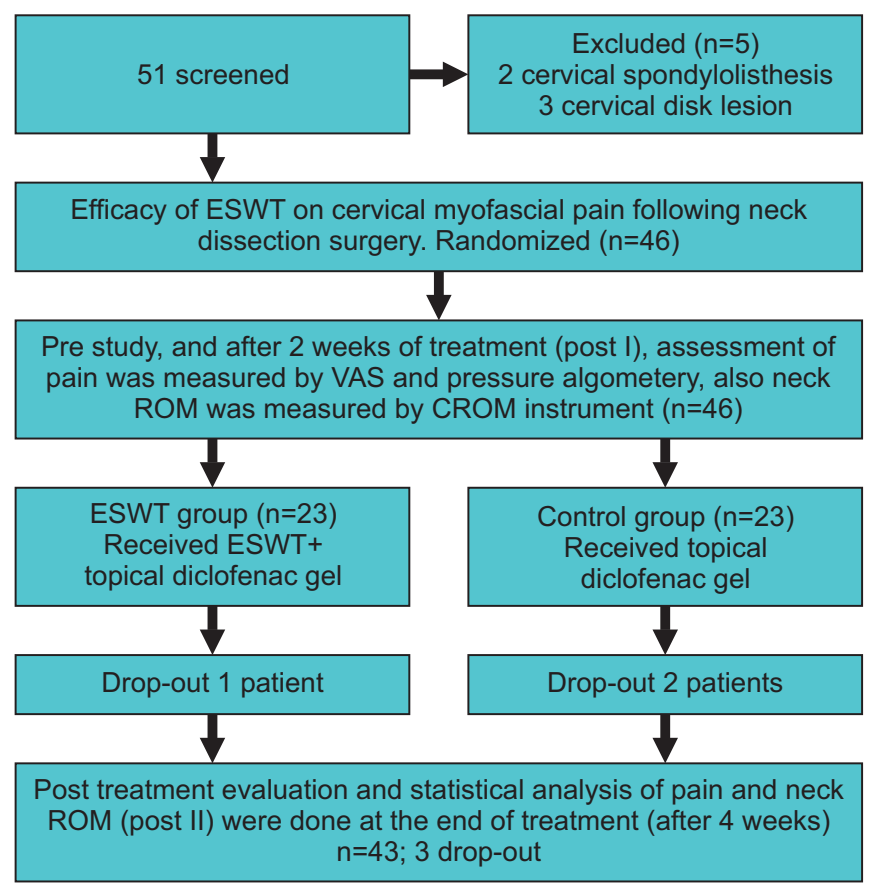

Fig. 1. Participants' flowchart. ESWT, extracorporeal shockwave therapy; VAS, visual analog scale; ROM, range of motion; CROM, cervical range of motion. 
Table 1. Basic characteristics of all participants

\begin{tabular}{lrcc}
\hline \multicolumn{1}{|c}{ Characteristic } & $\begin{array}{c}\text { Study } \\
\text { group }\end{array}$ & $\begin{array}{c}\text { Control } \\
\text { group }\end{array}$ & p-value \\
\hline Age (yr) & $48.8 \pm 7.5$ & $50.8 \pm 6.3$ & 0.35 \\
Weight (kg) & $84.1 \pm 3.7$ & $83.4 \pm 3.3$ & 0.48 \\
\hline Height (cm) & $173.9 \pm 4.4$ & $174.2 \pm 2.9$ & 0.80 \\
Time since surgery (mo) & $13.13 \pm 1.28$ & $13.28 \pm 1.34$ & 0.71 \\
Onset of pain (mo) & $6.27 \pm 1.03$ & $6.58 \pm 1.08$ & 0.44 \\
Sex & & & 0.55 \\
$\quad$ Male & $7(32)$ & $5(24)$ & \\
Female & $15(68)$ & $16(76)$ & \\
Affected side & & & 0.42 \\
$\quad$ Right & $18(82)$ & $15(71)$ & \\
\hline Left & $4(18)$ & $6(29)$ & \\
Diagnosis for surgery & & & 0.37 \\
\hline Oral/oropharynx & $10(46)$ & $7(33)$ & \\
Larynx/hypopharynx & $8(36)$ & $12(57)$ & \\
$\quad$ Thyroid & $4(18)$ & $2(10)$ & \\
\hline
\end{tabular}

Values are presented as mean \pm standard deviation or number (\%).

\section{Between group comparisons}

A significant increment of neck rotation, and side bending ROM in both directions away and toward the operated side with a marked improvement of PPT was reported at post I and post II in the ESWT group compared to the control group ( $p>0.001)$. Moreover, there was a significant decrease in VAS in the ESWT group at post I and post II compared to the control group ( $p>0.001)$.

\section{Within group comparison}

Statistical improvements were noted in the rotation and side bending ROM in both directions away and toward the operated side, and also in the PPT in the ESWT group at post II in comparison with the pre-treatment and post I ( $<<0.001)$; furthermore, there was a significant amelioration in the rotation and side bending ROM in both directions, and the PPT at post I compared with that of pre-treatment $(\mathrm{p}<0.001)$. There was a statistical diminution of the VAS score in the ESWT group at post II compared to pre-treatment and post I ( $\mathrm{p}<0.001)$; and also, in the VAS score at post I in comparison with pre-treatment $(\mathrm{p}<0.001)$.

There was no statistical difference in the rotation and side bending ROM in both directions and in the PPT between pre-treatment and post I ( $p>0.05)$ in the control

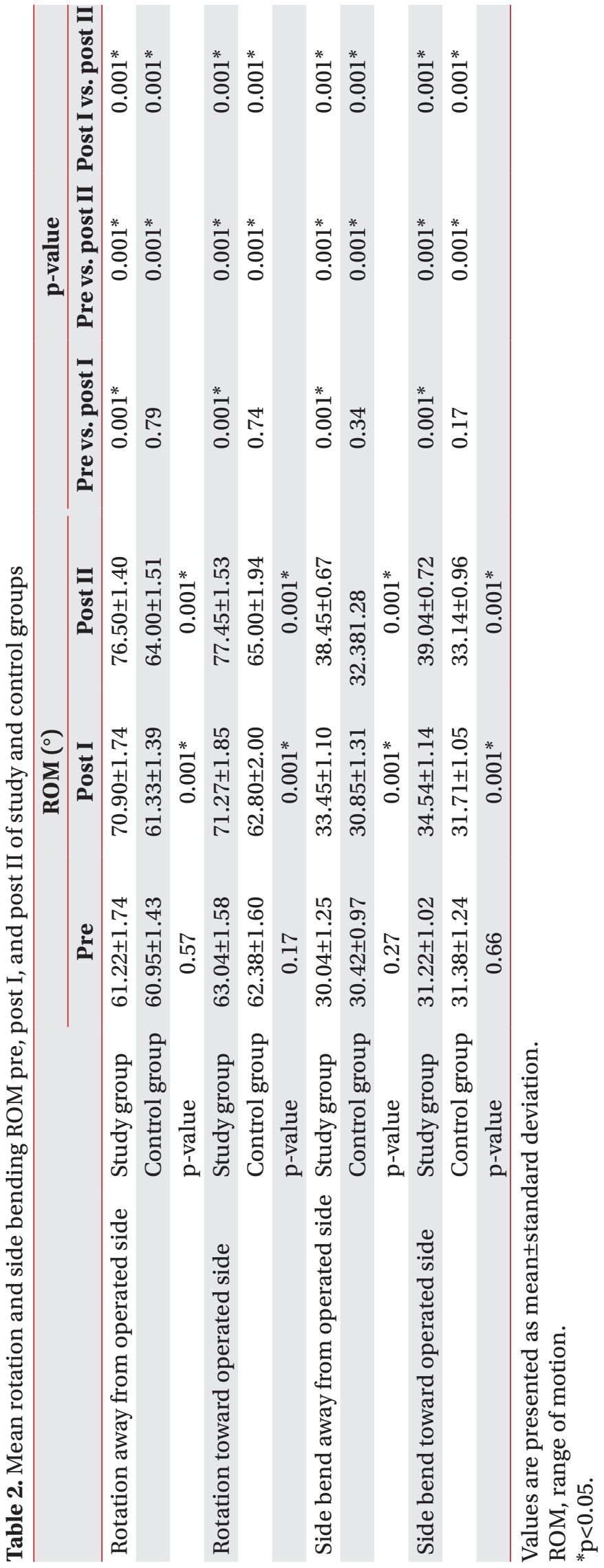

www.e-arm.org 
Table 3. Mean VAS and PPT pre, post I, and post II of study and control groups

\begin{tabular}{llcccccc}
\hline & & \multirow{2}{*}{ Pre } & Post I & \multirow{2}{*}{ Post II } & \multicolumn{3}{c}{ p-value } \\
\cline { 6 - 8 } & & & & Pre vs. post I & Pre vs. post II Post I vs. post II \\
\hline VAS & Study group & $6.31 \pm 0.77$ & $3.63 \pm 0.58$ & $1.72 \pm 0.63$ & $0.001^{*}$ & $0.001^{*}$ & $0.001^{*}$ \\
& Control group & $6.14 \pm 0.91$ & $4.47 \pm 0.92$ & $3.04 \pm 0.86$ & $0.001^{*}$ & $0.001^{*}$ & $0.001^{*}$ \\
& p-value & 0.5 & $0.001^{*}$ & $0.001^{*}$ & & & \\
\multirow{2}{*}{ PPT $(\mathrm{kg})$} & Study group & $2.80 \pm 0.22$ & $3.95 \pm 0.25$ & $4.98 \pm 0.37$ & $0.001^{*}$ & $0.001^{*}$ & $0.001^{*}$ \\
& Control group & $2.87 \pm 0.21$ & $2.91 \pm 0.24$ & $3.17 \pm 0.24$ & 0.28 & $0.001^{*}$ & $0.001^{*}$ \\
& p-value & 0.36 & $0.001^{*}$ & $0.001^{*}$ & & & \\
\hline
\end{tabular}

Values are presented as mean \pm standard deviation.

VAS, visual analog scale; PPT, pressure pain threshold.

${ }^{*} \mathrm{p}<0.05$.

group; however, there was a statistical increase in the rotation and side bending ROM in both directions and also in the PPT at post II in comparison with pre-treatment and post $I(p<0.001)$. There was a statistical decrease in the VAS score in the control group at post II compared with that at pre-treatment and post I ( $\mathrm{p}<0.001)$; as well as in the VAS score at post I in comparison with pre-treatment $(p<0.001)$.There were no adverse effects reported during the trial.

\section{DISCUSSION}

ESWT has been known as one of the most effective modalities for different musculoskeletal disorders including tendinitis, plantar fasciitis, chronic pelvic pain and rotator cuff disease [28]. In this study, a significant increase in neck rotation was noted in the ESWT group as the percentage changes in neck rotation away from and toward the operated side were $15.8 \%$ and $13.05 \%$ at post I and increased to $24.95 \%$ and $22.85 \%$ at post II, respectively. A significant increase in side bending was reported as the percentage changes in side bending away from and toward the operated side were $11.35 \%$ and $10.63 \%$ at post I and increased to $27.99 \%$ and $25.04 \%$ at post II, respectively. The PPT and VAS score assessments showed significant improvements as the percentage of perceived pain was decreased by $41.07 \%$ and $42.47 \%$ at post I and changed to $77.85 \%$ and $72.74 \%$ at post II, respectively. These outcomes may be due to the vertical mechanistic energy of ESWT applied to the firmly connected actinmyosin; furthermore, pain alteration may occur via the release of substance $P$ and nitric oxide.

ESWT has been evaluated in several different studies, and Ji et al. [29] evaluated a total of 4 sessions of ESWT $\left(0.056 \mathrm{~mJ} / \mathrm{mm}^{2}, 1,000\right.$ impulses, twice/week) in patients with MPS in the upper trapezius. The assessment by the VAS score and pressure algometry showed a significant difference as the percentages of improvement of the VAS score and PPT were $53.76 \%$ and $51.48 \%$, respectively. On the other hand, Jeon et al. [30] evaluated the aforementioned parameters in 3 sessions of ESWT $\left(0.10 \mathrm{~mJ} / \mathrm{mm}^{2}\right.$, 1,500 pulse, one session per week) and reported $72.88 \%$ and $83.23 \%$, respectively. Park et al. [31] compared different energy levels of ESWT $\left(0.210 \mathrm{~mJ} / \mathrm{mm}^{2}\right.$ vs. $0.068 \mathrm{~mJ} /$ $\mathrm{mm}^{2}, 1,500$ pulses, one session a week for 2 weeks) in 30 patients with MPS of the upper trapezius. The percentages of improvement of the VAS score and PPT at a higher intensity were $47.08 \%$ and $42.33 \%$, respectively, and $43.14 \%$ and $22.75 \%$, respectively, at a lower intensity.

The results of the previous studies support the findings of this study, reflecting significant improvement in pain intensity and threshold, the similarity between past studies and this study is using focused ESWT and the same methods of assessment, nevertheless, there is difference in the outcomes, the reason for this is not clear, it may be as a result of using different energy level in each study, another possible explanation for these results may be the lack of standardized ESWT parameters used for MPS management. Moreover, the previous studies evaluated ESWT on primary MPS, unlike this study which assessed MPS secondary to neck dissection surgery.

Gur et al. [32] compared one to three sessions of ESWT $\left(0.25 \mathrm{~mJ} / \mathrm{mm}^{2}, 1,000\right.$ impulses $)$ in 64 patients with MPS of the trapezius muscle. The assessments of pain, quality of life, and trigger points count showed significant improvements in both groups, while higher results were observed 
in the group receiving 3 sessions. In another study, the same authors evaluated 3 sessions of the same ESWT parameters versus 10 sessions of US in 66 patients with MPS in the trapezius using the previous methods of assessment. The results showed the efficacy of both modalities in MPS with a superiority of ESWT outcomes in pain management [33]. There are similarities between the two aforementioned studies and our study in terms of the ESWT parameters used; however, our study differs from theirs in that objective assessment methods were used in ours, unlike in theirs. Notwithstanding, the results of these studies coincided with our results as far as the efficacy of ESWT in pain management was concerned.

Furthermore, Hong et al. [34] made a comparison between 3 sessions of ESWT $\left(0.085-0.148 \mathrm{~mJ} / \mathrm{mm}^{2}, 2,000\right.$ impulses) and 3 sessions of TPI in quadratus lumborum using the VAS score and digital algometer for pain assessment. Both treatments showed significant improvements in the VAS score and pain threshold $(65 \%$ and $39.18 \%$ vs. $44.12 \%$ and $10.39 \%$, respectively). However, the ESWT achieved better results and was superior to TPI suggesting it is a more effective modality for MPS treatment. From all the previous studies, it can be concluded that efficiency of ESWT in MPS management is based on the underlying mechanism of ESWT in decreasing the level of muscle tension and the amelioration of localized ischemia in the regions of abnormal muscles shortening. Hence, it prevents the increase in metabolism and onset of an energy crisis, thereby allowing muscle relaxation which in turn increases muscle length and permits a free ROM. In addition, the ESWT eliminates the release of different pain inducing substances, and prevents pain induction caused by the extra excitation of nociceptors of muscles and the selective destruction of non-myelinated fibers.

In this study, better statistical results were reported in VAS, PPT, and neck ROM of the ESWT group than in the control group ( $p>0.001)$. However, this study was limited by a small sample size and the absence of shoulder pain and ROM assessments which could provide better statistical analyses. Further researches are recommended with larger sample sizes to evaluate and assure the efficacy of ESWT in MPS and to examine different intensities and frequencies in an attempt to reach the optimal doses in musculoskeletal disorders, as the absence of definite guidelines for ESWT standardized parameters is also considered a limitation. Also, the efficacy of the ESWT in improving the result measures are surmised exclusively depending on the past evaluation; hence, additional histological and molecular studies ought to have been conducted along with the current evaluation. Lack of previous studies on the impacts of ESWT on postsurgical myofascial pain is another limitation. We, therefore, recommend further studies to investigate the effects of shockwaves on MPS after different surgeries. Followup studies are also required to reveal ESWT effects in the long run.

In conclusion, ESWT is an effective and safe treatment modality for cervical MPS following neck dissection. These results demonstrated that ESWT could be an alternative treatment for cervical MPS following neck dissection.

\section{CONFLICT OF INTEREST}

No potential conflict of interest relevant to this article was reported.

\section{AUTHOR CONTRIBUTION}

Conceptualization: Aboelnour NH, Kamel FH, Basha M, Ezzat M. Methodology: Aboelnour NH, Ezzat M. Formal analysis: Kamel FH, Basha M, Aboelnour NH, Hewidy IM. Funding acquisition: None. Project administration: Aboelnour NH, Ezzat M. Visualization: Aboelnour NH, Kamel FH, Basha M, Hewidy IM. Writing - original draft: Aboelnour NH, Kamel FH, Basha M, Hewidy IM. Writing - review and editing: Hewidy IM, Ezzat M, Alsharidah A. Approval of final manuscript: all authors.

\section{REFERENCES}

1. Burusapat C, Jarungroongruangchai W, Charoenpitakchai M. Prognostic factors of cervical node status in head and neck squamous cell carcinoma. World J Surg Oncol 2015;13:51.

2. Pugazhendi SK, Thangaswamy V, Venkatasetty A, Thambiah $\mathrm{L}$. The functional neck dissection for lymph node neck metastasis in oral carcinoma. J Pharm Bioallied Sci 2012;4(Suppl 2):S245-7.

3. Ferlito A, Robbins KT, Shah JP, Medina JE, Silver CE, Al-Tamimi S, et al. Proposal for a rational classifica- 
tion of neck dissections. Head Neck 2011;33:445-50.

4. Van Wilgen CP, Dijkstra PU, van der Laan BF, Plukker JT, Roodenburg JL. Morbidity of the neck after head and neck cancer therapy. Head Neck 2004;26:785-91.

5. Guru K, Manoor UK, Supe SS. A comprehensive review of head and neck cancer rehabilitation: physical therapy perspectives. Indian J Palliat Care 2012;18:8797.

6. Sist T, Miner M, Lema M. Characteristics of postradical neck pain syndrome: a report of 25 cases. J Pain Symptom Manage 1999;18:95-102.

7. Bron C, Dommerholt JD. Etiology of myofascial trigger points. Curr Pain Headache Rep 2012;16:439-44.

8. Koca I, Boyaci A. A new insight into the management of myofascial pain syndrome. Gaziantep Med J 2014;20107-12.

9. Han TR, Bang MS. Myofascial pain. In: Kang YK, editor. Rehabilitation medicine. 3rd ed. Seoul, Korea: Koonja; 2008. p. 887-96.

10. Cardoso LR, Rizzo CC, de Oliveira CZ, dos Santos CR, Carvalho AL. Myofascial pain syndrome after head and neck cancer treatment: prevalence, risk factors, and influence on quality of life. Head Neck 2015;37:1733-7.

11. Desai MJ, Saini V, Saini S. Myofascial pain syndrome: a treatment review. Pain Ther 2013;2:21-36.

12. Schmitz C, Csaszar NB, Rompe JD, Chaves H, Furia JP. Treatment of chronic plantar fasciopathy with extracorporeal shock waves (review). J Orthop Surg Res 2013;8:31.

13. Ioppolo F, Rompe JD, Furia JP, Cacchio A. Clinical application of shock wave therapy (SWT) in musculoskeletal disorders. Eur J Phys Rehabil Med 2014;50:217-30.

14. Speed C. A systematic review of shockwave therapies in soft tissue conditions: focusing on the evidence. $\mathrm{Br}$ J Sports Med 2014;48:1538-42.

15. Gleitz M. Myofascial syndrome and trigger points. Heilbronn, Germany: Level 10 Book; 2011.

16. Ramon S, Gleitz M, Hernandez L, Romero LD. Update on the efficacy of extracorporeal shockwave treatment for myofascial pain syndrome and fibromyalgia. Int J Surg 2015;24(Pt B):201-6.

17. Schmitz C, Csaszar NB, Milz S, Schieker M, Maffulli $\mathrm{N}$, Rompe JD, et al. Efficacy and safety of extracorporeal shock wave therapy for orthopedic conditions: a systematic review on studies listed in the PEDro database. Br Med Bull 2015;116:115-38.

18. Gleitz M, Hornig K. Trigger points: Diagnosis and treatment concepts with special reference to extracorporeal shockwaves. Orthopade 2012;41:113-25.

19. Suputtitada A. Novel approaches for chronic pain. Int J Phys Med Rehabil 2016;4:350.

20. Saggini R, Di Stefano A, Saggini A, Bellomo RG. Clinical application of shock wave therapy in musculoskeletal disorders: part II related to myofascial and nerve apparatus. J Biol Regul Homeost Agents 2015;29:77185.

21. Wang CJ. Extracorporeal shockwave therapy in musculoskeletal disorders. J Orthop Surg Res 2012;7:11.

22. Notarnicola A, Moretti B. The biological effects of extracorporeal shock wave therapy (ESWT) on tendon tissue. Muscles Ligaments Tendons J 2012;2:33-7.

23. Zhang D, Kearney CJ, Cheriyan T, Schmid TM, Spector M. Extracorporeal shockwave-induced expression of lubricin in tendons and septa. Cell Tissue Res 2011;346:255-62.

24. Romeo P, Lavanga V, Pagani D, Sansone V. Extracorporeal shock wave therapy in musculoskeletal disorders: a review. Med Princ Pract 2014;23:7-13.

25. Hausdorf J, Lemmens MA, Heck KD, Grolms N, Korr H, Kertschanska S, et al. Selective loss of unmyelinated nerve fibers after extracorporeal shockwave application to the musculoskeletal system. Neuroscience 2008;155:138-44.

26. Travell JG, Simons DG, Simons LS. Myofascial pain and dysfunction: the trigger point manual (Vol. 1). 2nd ed. Baltimore, MD: Lippincott Williams \& Wilkins; 1999.

27. Faul F, Erdfelder E, Buchner A, Lang AG. Statistical power analyses using $G^{*}$ Power 3.1: tests for correlation and regression analyses. Behav Res Methods 2009;41:1149-60.

28. Suputtitada A. Update of extracorporeal shockwave therapy in myofascial pain syndrome. Int Phys Med Rehab J 2017;1:00019.

29. Ji HM, Kim HJ, Han SJ. Extracorporeal shock wave therapy in myofascial pain syndrome of upper trapezius. Ann Rehabil Med 2012;36:675-80.

30. Jeon JH, Jung YJ, Lee JY, Choi JS, Mun JH, Park WY, et al. The effect of extracorporeal shock wave therapy on myofascial pain syndrome. Ann Rehabil Med 
2012;36:665-74.

31. Park KD, Lee WY, Park MH, Ahn JK, Park Y. High- versus low-energy extracorporeal shock-wave therapy for myofascial pain syndrome of upper trapezius: a prospective randomized single blinded pilot study. Medicine (Baltimore) 2018;97:e11432.

32. Gur A, Koca I, Karagullu H, Altindag O, Madenci E, Tutoglu A, et al. Comparison of the effectiveness of two different extracorporeal shock wave therapy regimens in the treatment of patients with myofascial pain syndrome. Arch Rheumatol 2014;29:186-93.
33. Gur A, Koca I, Karagullu H, Altindag O, Madenci E. Comparison of the efficacy of ultrasound and extracorporeal shock wave therapies in patients with myofascial pain syndrome: a randomized controlled study. J Musculoskelet Pain 2013;21:210-6.

34. Hong JO, Park JS, Jeon DG, Yoon WH, Park JH. Extracorporeal shock wave therapy versus trigger point injection in the treatment of myofascial pain syndrome in the quadratus lumborum. Ann Rehabil Med 2017;41:582-8. 